\title{
COMMUNITY RATING AND EQUALISATION ${ }^{1}$
}

\author{
Walther NeuHaus \\ University of Copenhagen and MIRA Consultants Ltd. (Sydney)
}

\begin{abstract}
Several countries have made community rating mandatory for certain lines of insurance, particularly health insurance. This paper offers a theoretical solution to the problem of designing equalisation schemes to support community rating in a market where different insurers are selling different benefit plans. The criterion chosen is that an equalisation scheme should minimise the opportunities for arbitrage between insurers, which community rating otherwise would generate. Several possible measures of arbitrage are presented, and the optimal schemes are compared against data from Australian health insurers. Finally, the approach is extended to partial community rating, for example unisex rating.
\end{abstract}

\section{KEYWORDS}

Arbitrage; Community rating; Credibility theory; Equalisation; Health insurance; Risk adjustment.

\section{INTRODUCTION}

Private insurers in Australia are required by law to practise community rating. They are not allowed to differentiate their contribution rates by the age, sex or any other criteria of the applicant (including ill-health). The only allowable discrimination is between single members and family members, the contribution rate for a family being twice the contribution rate for a single person. While community rating in this way imposes a strong constraint on the rating structure of private health insurers, there is no explicit regulation governing rate levels.

Two tiers of benefit plans are offered by private health insurers in Australia: the basic plan, which is determined by the Government and common to all insurers, and supplementary plans, which may differ between insurers. The basic plan provides for private accommodation in public hospitals as well as some medical expenses, while supplementary plans normally provide for accommodation in private hospitals. A large majority of the privately insured hold both the basic and a supplementary plan.

The reason given for mandatory community rating is one of social equity, i.e. the wish to ensure that private health insurance remains affordable for all who choose

\footnotetext{
${ }^{1}$ Paper presented at the Oberwolfach Conference on Risk Theory, 18th to 24th September 1994.
} 
to buy it. All Australian residents are also covered by the public health care system (Medicare), the introduction of which has reduced the privately insured population significantly.

While it is debatable whether community rating actually achieves its goal (higher enrolment through universal affordability), there is general agreement that community rating can only work if enforced by legislation, or in a monopoly market. For a thorough discussion of community rating in voluntary health insurance, see Macintyre (1962).

It is well known that mandatory community rating in a competitive market can lead to market instability and adverse selection against some insurers. Insurers with a large proportion of elderly persons are particularly disadvantaged under community rating, as the cost of providing health insurance increases rapidly with a person's age. In Australia, the average drawing rate of persons aged over 65 is five times that of persons under 65. This is shown in Figure 1.1 (Health insurers refer to the expected benefit cost of a person as his/her drawing rate; actuaries commonly use the term pure premium).

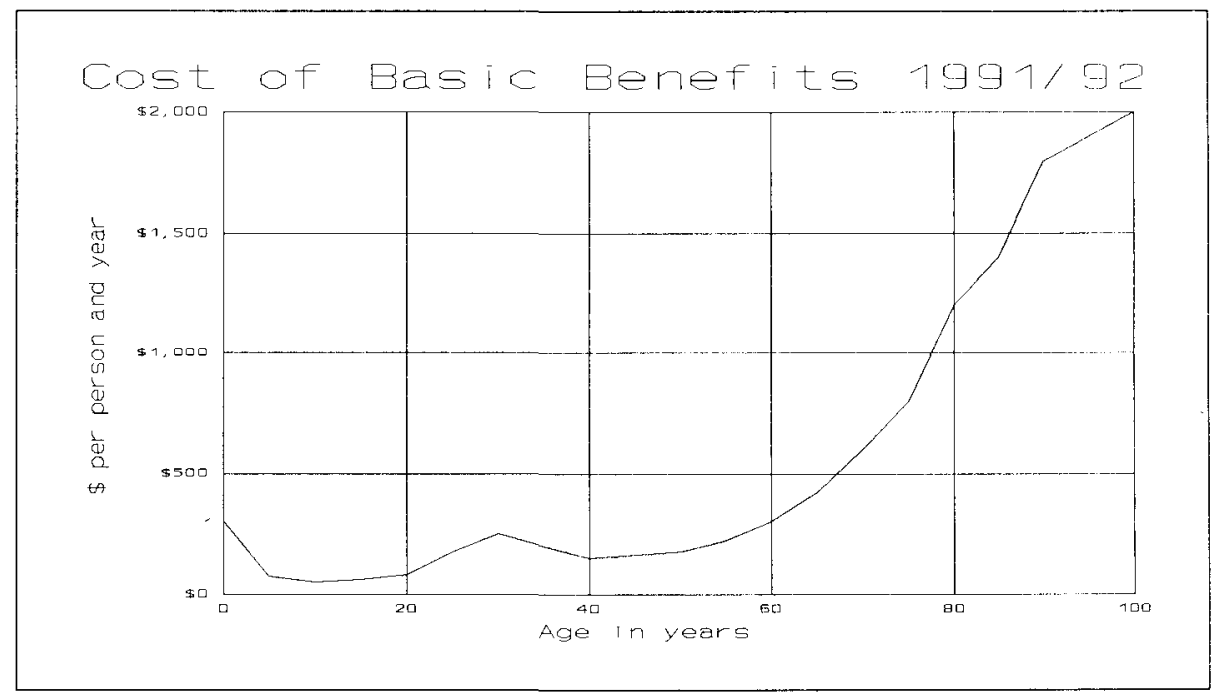

Figure 1.1

Several countries have implemented or are proposing to implement equalisation schemes which support community rating by cross-subsidies between insurers with a young age profile and insurers with an old age profile. De Wit and van Eeghen (1984) as well as Gregorius (1987) describe the situation in the Netherlands. In Australia, an equalisation scheme was introduced in 1989 and modified in 1995. The Department of Health of the Republic of Ireland (1994) has proposed an equalisation scheme to support community rating under the EC Third Directive on Non-Life Insurance. 
Broadly speaking, the objective of all equalisation schemes is to level the playing field between insurers with different membership profiles, and to reduce the incentive for insurers to engage in "predatory marketing" or "cherry-picking".

Equalisation is straightforward in a market where all insurers offer just one standard benefit plan: all one has to do is to allocate to each insurer the global average cost per person, times the number of persons it has insured.

This paper provides a theoretical framework for the design of an equalisation scheme in a market where different insurers offer different benefit plans.

The concept of arbitrage is invoked to measure the degree of mismatch between the benefit plans and the community-rated contribution rates of different insurers. The optimal equalisation scheme is the one which minimises the possibility for arbitrage, suitably defined.

In section 2 we discuss the notion of equity (fairness) between insurers and introduce the concept of arbitrage. Section 3 presents a minimum arbitrage scheme which minimises arbitrage opportunities between an insurer and the market average. In section 4 we develop a more elaborate minimum arbitrage scheme, which minimises arbitrage opportunities between all health insurers. Section 5 presents a study of data from Australia ; in that section we also compare the optimal schemes with a simplified variant of the scheme used in Australia since 1989.

Section 6 looks at an alternative measure of arbitrage. In section 7 we extend the theory to cover partial community rating, for example unisex rating. Some concluding remarks are given in section 8 .

\section{EQUITY BETWEEN HEALTH INSURERS}

It seems self-evident that while an equalisation should equalise variations in cost which are the result of different membership profiles, it should not equalise variation in cost which can be ascribed to different levels of benefits. In what follows we justify this argument with reference to arbitrage. We will argue that an equalisation scheme provides equity (fairness) between health insurers if it eliminates, or at least minimises the incentive for arbitrage which community rating generates.

An opportunity for arbitrage between two insurers exists when there is a mismatch between the benefit levels they offer and the contribution rates they have to charge to support their benefit levels. Let us consider some examples.

If two insurers differ in their membership profile but offer the same level of benefits, differences in their cost will reflect the difference in membership profiles; the insurer with the highest proportion of elderly members will have the highest cost. If there is no equalisation, the difference in cost will have to be reflected in different contribution rates. This would allow mobile members of the higher-cost insurer to switch to the lower-cost insurer and receive the same level of benefits for a smaller contribution. This is a form of arbitrage.

As an aside we note that the most mobile members tend to be the younger members. Thus, if arbitrage between two insurers occurs along the lines sketched above, then it will tend to increase the incentive for arbitrage as the difference in the age profile grows. 
If two insurers have the same membership profile and different levels of benefit, their cost will reflect the differences in benefits paid and will have to be reflected in different contribution rates if there is no equalisation. Thus for a member of one insurer, switching to the other insurer would involve a trade-off between contributions and benefits and there is no opportunity for arbitrage. We conclude that different levels of benefits need not be equalised.

Indeed, equalising different levels of benefits can introduce an arbitrage opportunity, as can be seen from the following argument. If the cost of different benefit levels are equalised, two insurers with different benefit levels may be induced to charge similar contribution rates. This would allow mobile members of the insurer with lower benefits to "upgrade" to higher benefits without a commensurate increase in their contribution; again, a form of arbitrage.

As can be seen from the discussion above, community rating without any form of equalisation generates arbitrage opportunities by preventing insurers from charging new applicants their true pure premium. Even if each insurer at the outset had a perfectly balanced membership, aggressive marketing by some insurers can lead to an imbalance which may be self-reinforcing and destabilise the market.

Thus is can be argued that if a government imposes community rating on a competitive industry (health insurance or otherwise), it has an obligation to support community rating by some form of equalisation. We describe an equalisation scheme as fair if it eliminates, or at least minimises, the opportunities for arbitrage which mandatory community rating otherwise would generate.

The reader should note that in this paper, arbitrage is considered only in terms of the pure benefit cost faced by the insurers. For the insured, there may be arbitrage opportunities generated by other factors, for example different expense levels or investment strategies. There is no need to eliminate those arbitrage opportunities, as they are not a direct result of government policy.

\section{MINIMISING ARBITRAGE AGAINST THE MARKET AVERAGE}

In this section we develop a class of equalisation schemes which minimises the opportunity for arbitrage between an insurer and the market average. We begin by introducing some notation.

Denote the insurers operating in the market by $i=1, \ldots, I$. Let $\mathrm{X}$ denote a finite partition of the insured population into homogeneous risk classes. It is helpful to visualise $\mathrm{X}$ as a collection of age groups, but the partition may reflect other factors that affect cost as well. For an insurer $i \in\{1, \ldots, I\}$ and a risk class $x \in \mathrm{X}$, we define the following quantities:

$n_{i}(x)=$ the number of person years covered during a given year;

$B_{i}(x)=$ the amount of benefits paid or incurred during the same period.

Corresponding symbols without the argument $(x)$ denote the corresponding quantity summed across all age groups. Likewise, corresponding symbols with the subscript $i$ omitted denote the corresponding quantity summed across all insurers. Thus, for instance, $n_{i}$ denotes the number of persons covered by insurer $i$, while $n(x)$ denotes the number of persons in risk class $x$ in the entire insured population. 
Denote by $d_{i}(x)$ the drawing rate incurred by insurer $i$ in insuring a person in class $x$, that is

$$
\mathrm{E} B_{i}(x)=n_{i}(x) d_{i}(x) .
$$

The average drawing rate incurred by all insurers in relation to class $x$ is then

$$
d(x)=[n(x)]^{-1} \sum_{i=1}^{I} n_{i}(x) d_{i}(x)
$$

The overall drawing rate incurred by insurer $i$ across all classes is

$$
d_{i}=n_{i}^{-1} \sum_{x \in \mathrm{X}} n_{i}(x) d_{i}(x)
$$

and the overall drawing rate incurred by all insurers across all classes is

$$
d=n^{-1} \sum_{i=1}^{l} \sum_{x \in \mathrm{X}} n_{i}(x) d_{i}(x) .
$$

In the absence of equalisation, the contribution rates charged by insurer $i$ are essentially determined by $d_{i}$, while the average contribution rate of all insurers is essentially determined by $d$. We say that class $x \in X$ has an arbitrage opportunity between insurer $i$ and the market, if

$$
d_{i}-d \neq d_{i}(x)-d(x) \text {. }
$$

Thus an arbitrage opportunity exists whenever there is a mismatch between the difference in contribution rates and the difference in benefits. In order to get an overall measure of arbitrage opportunities present in the market, we define the measure

$$
Q\left(d_{1}, \ldots d_{l}\right)=\sum_{i=1}^{I} \sum_{x \in \mathrm{X}} v_{i}(x)\left(d_{i}-d-d_{i}(x)+d(x)\right)^{2},
$$

where $\left\{v_{i}(x) \mid i=1, \ldots, \quad l ; x \in \mathrm{X}\right\}$ is a set of arbitrary, fixed non-negative weights.

Under an equalisation scheme there is a zero-sum reallocation of costs, and the overall drawing rate $d_{i}$ of insurer $i$ is replaced by a quantity $\tilde{d}_{i}$, the post-equalisation unit cost.

Thus in order to minimise arbitrage by equalisation, one must solve the constrained minimisation problem

$$
\text { Minimise } \sum_{i=1}^{I} \sum_{x \in X} v_{i}(x)\left(\tilde{d}_{i}-d-d_{i}(x)+d(x)\right)^{2}
$$

with respect to $\tilde{d}_{1}, \ldots, \tilde{d}_{1}$,

subject to $\sum_{i=1}^{\prime} n_{i} \tilde{d}_{i}=n d$. 
The side condition ensures that the equalisation scheme is balanced, on average. We refer to the side condition as the balancing constraint.

\section{Theorem 3.1}

The minimum arbitrage scheme against the criterion (3.6) is given by the allocation

$$
\tilde{d}_{k}=d+\Delta_{k}-\frac{n_{k}}{v_{k}}\left[\sum_{i=1}^{l} \frac{n_{i}^{2}}{v_{i}}\right]^{-1} \sum_{i=1}^{l} n_{i} \Delta_{i}
$$

where $v_{k}=\sum_{x \in \mathrm{X}} v_{k}(x)$ and

$$
\Delta_{i}=\sum_{x \in X} \frac{v_{i}(x)}{v_{i}}\left(d_{i}(x)-d(x)\right)
$$

Proof: Lagrange minimisation. One must determine the solution of the $I$ equations

(3.11) $\frac{\partial}{\partial \tilde{d}_{k}}\left[\frac{1}{2} \sum_{i=1}^{l} \sum_{x \in X} v_{i}(x)\left(\tilde{d}_{i}-d-d_{i}(x)+d(x)\right)^{2}+\lambda\left(\sum_{i=1}^{l} n_{i} \tilde{d}_{i}-n d\right)\right]=0, \forall k$,

which turns out to be

$$
\tilde{d}_{k}=d+\Delta_{k}-\lambda \frac{n_{k}}{v_{k}}
$$

The constraint $\sum_{i=1}^{I} n_{i} \tilde{d}_{i}=n d$ is then applied to yield

$$
\lambda=\left[\sum_{i=1}^{l} \frac{n_{i}^{2}}{v_{i}}\right]^{-1} \sum_{i=1}^{l} n_{i} \Delta_{i} .
$$

This proves the theorem.

The last term in (3.9) is obviously a balancing correction, ensuring that the balancing constraint is observed.

\section{Corollary 3.2.}

In the special case where $v_{i}(x)=c \cdot n_{i}(x)$ with an arbitrary constant $c>0$, we obtain the following minimum arbitrage allocation:

$$
\tilde{d}_{k}=d+\sum_{x \in \mathrm{X}} p_{k}(x)\left(d_{k}(x)-d(x)\right)
$$


where $p_{k}(x)=n_{k}(x) / n_{k}$ is the proportion of class $x$ in the membership of insurer $k$.

Proof: Simple substitution. Note that in this case, $\lambda=0$.

QED

\section{Remark 3.3.}

The choice $v_{i}(x)=c \cdot n_{i}(x)$ obviously makes sense. It means that the squared arbitrage terms in (3.8) are weighted in proportion to the number of persons exposed to the arbitrage opportunity in question.

\section{Remark 3.4.}

In our discussion so far, we have discussed arbitrage only in terms of pure premiums, or expected values. In order to develop a fully operational equalisation scheme, one has to replace the drawing rates $d_{i}(x)$ by suitable estimates; let us denote the estimates by $d_{i}^{*}(x)$. One would normally also want to balance the equalisation against the actual claims cost $B$ of all insurers, rather than the expected claims cost $n d$. This will be automatically the case if the estimates balance, i.e.

$$
n d^{*}=\sum_{i=1}^{l} \sum_{x \in \mathrm{X}} n_{i}(x) d_{i}^{*}(x)=B .
$$

Neuhaus (1995) shows how credibility estimators can be corrected to balance.

Alternatively one could estimate each $d_{i}(x)$ by the empirical drawing rate

$$
\hat{d}_{i}(x):=D_{i}(x)=B_{i}(x) / n_{i}(x) .
$$

It is easy to verify that the estimators $\hat{d}_{i}(x)$ balance. They have the additional advantage of being easy to explain to non-mathematical people. Inserting empirical drawing rates into the scheme defined in (3.14), one obtains the scheme

$$
\tilde{D}_{k}=D+\sum_{x \in \mathrm{X}} p_{k}(x)\left(D_{k}(x)-D(x)\right)
$$

where $D(x)=B(x) / n(x)$ is the average drawing rate of class $x$ and $D=B / n$ is the overall average drawing rate. The equalisation scheme defined by $(3.17)$ is called a composition-based scheme in MIRA (1993, 1994).

Thomson (1994) suggested the use of robust estimation for the drawing rates. This is entirely possible, but a little more effort will be required to ensure that the estimates balance. Simple grossing up of the robust estimates with a constant factor is one option.

\section{Remark 3.5.}

The equalisation formula (3.17) can be derived heuristically without any reference to arbitrage. To see this, decompose the drawing rate of insurer $k$ into three 
components as follows :

$$
\begin{aligned}
D_{k} & =D \\
& +\sum_{x \in \mathrm{X}} p_{k}(x)\left(D_{k}(x)-D(x)\right) \quad\left(=: \Delta_{k}(D)\right) \\
& +\sum_{x \in \mathrm{X}} D(x)\left(p_{k}(x)-p(x)\right) \quad\left(=: \Delta_{k}(p)\right)
\end{aligned}
$$

The three components can be called the average drawing rate $D$, the benefit component $\Delta_{k}(D)$ of insurer $k$, and the profile component $\Delta_{k}(p)$ of insurer $k$. In keeping with the intuitively obvious notion that differences in profile should be equalised but not differences in benefit levels, one can eliminate the profile component and equalisation scheme (3.17) appears.

\section{MINIMISING ARBITRAGE BETWEEN HEALTH INSURERS}

While the measure of arbitrage presented in the previous section leads to a neat and simple formula for the minimum arbitrage scheme, the underlying assumption that arbitrage occurs only between an insurer and the market average may be unrealistic. In reality, people insured with one insurer are able to check out the contribution rates of any number of its competitors, and move their policy if there is a mismatch between the contribution rates and the benefits between their current insurer and any one of its competitors. In this section we develop an equalisation scheme which minimises the opportunity for inter-insurer arbitrage.

Denote by $\tilde{d}_{i}$ the post-equalisation unit cost of an insurer $i$, given any specified equalisation scheme. For two insurers $i, j \in\{1, \ldots, I\}$, we say that people in risk class $x \in \mathrm{X}$ have an opportunity for arbitrage if

$$
\tilde{d}_{i}-\tilde{d}_{j} \neq d_{i}(x)-d_{j}(x) \text {. }
$$

For each $x \in X$, define a symmetric matrix $V(x)$ of fixed, non-negative weights,

$$
\mathrm{V}(x)=\left[\begin{array}{ccc}
\nu_{11}(x) & \ldots & \nu_{1 /}(x) \\
\vdots & \ldots & \vdots \\
v_{11}(x) & \ldots & v_{1 /}(x)
\end{array}\right]
$$

In order to get an overall measure of arbitrage opportunities present in the market, we define the measure

$$
Q\left(\tilde{d}_{1}, \ldots \tilde{d}_{l}\right)=\sum_{i=1}^{I} \sum_{j=1}^{I} \sum_{x \in \mathrm{X}} v_{i j}(x)\left(\tilde{d}_{i}-\tilde{d}_{j}-d_{i}(x)+d_{j}(x)\right)^{2}
$$

Note that the weighted sum (4.3) involves double counting of the off-diagonal terms, while the diagonal terms are zero. This has been done to simplify the subsequent algebraic bookkeeping. Double counting only changes the measure by a constant factor without affecting the optimal solution. 
In order to minimise the opportunity for arbitrage by equalisation, one must solve the constrained minimisation problem

$$
\begin{aligned}
& \text { Minimise } \sum_{i=1}^{I} \sum_{j=1}^{I} \sum_{x \in \mathrm{X}} v_{i j}(x)\left(\tilde{d}_{i}-\tilde{d}_{j}-d_{i}(x)+d_{j}(x)\right)^{2} \\
& \text { subject to } \sum_{i=1}^{I} n_{i} \tilde{d}_{i}=n d .
\end{aligned}
$$

The side condition ensures that the equalisation scheme is balanced, on average.

\section{Theorem 4.1}

The minimum arbitrage scheme against the criterion (4.3) is given by the set of linear equations

$$
v_{k} \tilde{d}_{k}=\sum_{i=1}^{I} v_{k i} \tilde{d}_{i}+\sum_{x \in \mathrm{X}} v_{k}(x) d_{k}(x)-\sum_{x \in \mathrm{X}} \sum_{i=1}^{I} v_{k i}(x) d_{i}(x)
$$

$$
\begin{aligned}
\text { for } k & =1, \ldots, I, \text { and } \\
n d & =\sum_{i=1}^{I} n_{i} \tilde{d}_{i},
\end{aligned}
$$

where

$$
\begin{aligned}
& v_{k}=\sum_{i=1}^{l} \sum_{x \in \mathrm{X}} v_{k i}(x), \\
& v_{k i}=\sum_{x \in \mathrm{X}} v_{k i}(x), \\
& v_{k}(x)=\sum_{i=1}^{I} v_{k i}(x) .
\end{aligned}
$$

Proof: Lagrange minimisation. Equating the partial derivatives of $\frac{1}{4} Q\left(\tilde{d}_{1}, \ldots, \tilde{d}_{i}\right)+\lambda\left(\sum_{i=1}^{\prime} n_{i} \tilde{d}_{i}-n d\right)$ with respect to $\tilde{d}_{1}, \ldots, \tilde{d}_{l}$ to zero yields (4.7) $\quad v_{k} \tilde{d}_{k}=\sum_{i=1}^{l} v_{k i} \tilde{d}_{i}+\sum_{x \in \mathrm{X}} v_{k}(x) d_{k}(x)-\sum_{x \in \mathrm{X}} \sum_{i=1}^{l} v_{k i}(x) d_{i}(x)-\lambda n_{k}$,

for $k=1, \ldots, I$. Summing (4.7) across all values of $k$ yields $\lambda=0$. Thus the balancing constraint cannot be effectively eliminated and we have to be content with the implicit formula (4.5).

QED 


\section{Corollary 4.2.}

If the weighting matrix $\mathrm{V}=\sum_{x \in \mathrm{X}} \mathrm{V}(x)$ satisfies the condition

$$
v_{i j}=c \cdot n_{i} n_{j}
$$

with an arbitrary constant $c>0$, then the minimum arbitrage scheme against the criterion (4.3) has an explicit solution, which is

$$
\tilde{d}_{k}=d+\sum_{x \in \mathrm{X}} \frac{v_{k}(x)}{v_{k}} d_{k}(x)-\sum_{x \in \mathrm{X}} \sum_{i=1}^{l} \frac{v_{k i}(x)}{v_{k}} d_{i}(x) .
$$

Proof : Under the condition of the corollary, the balancing constraint implies

$$
\sum_{i=1}^{I} v_{k i} \tilde{d}_{i}=c \cdot n_{k} \sum_{i=1}^{I} n_{i} \tilde{d}_{i}=c \cdot n_{k} n d=v_{k} d .
$$

QED

The condition of corollary 4.2 is sensible. In using weights of the form (4.8) the aggregate weight one assigns to arbitrage in (4.3) between any two insurers is proportional to the product of their market shares; one could also say that for any given insurer $i$, the seriousness of arbitrage against another insurer $j$ is proportional to the other insurer's market share. Thus the condition (4.8) formalises the intuitive feeling that arbitrage opportunities should be taken most seriously when the exposed insurers have a large share of the market.

\section{Remark 4.3.}

In the general formula (4.5) one can write

$$
\tilde{d}_{k}=\tilde{d}^{(k)}+\sum_{x \in \mathrm{X}} \frac{v_{k}(x)}{v_{k}}\left(d_{k}(x)-d^{(k)}(x)\right),
$$

where

$$
\begin{aligned}
& \tilde{d}^{(k)}=\sum_{i=1}^{I} \frac{v_{k i}}{v_{k}} \tilde{d}_{i}, \\
& d^{(k)}(x)=\sum_{i=1}^{I} \frac{v_{k i}(x)}{v_{k}(x)} d_{i}(x) .
\end{aligned}
$$

Note the formal similarity between (4.11) and (3.14). The current formula is more sophisticated than (3.14) in that the averages applied to each insurer, depend on the insurer and the weighting of arbitrage opportunities between that insurer and any of its competitors. In contrast, $(3.14)$ is based on the premise that only market averages matter. 


\section{Remark 4.4.}

The system of equations in (4.5) can be written in matrix form as

$$
[\operatorname{diag}(\mathrm{V} \cdot \mathbf{1})-\mathrm{V}] \tilde{\mathrm{d}}=\sum_{x \in \mathrm{X}}[\operatorname{diag}(\mathrm{V}(x) \cdot \mathbf{1})-\mathrm{V}(x)] \mathrm{d}(x),
$$

where $\tilde{\mathrm{d}}=\left[\tilde{d}_{1}, \ldots,\left.\tilde{d}_{l}\right|^{T}, \mathrm{~d}(x)=\left[d_{1}(x), \ldots, d_{l}(x)\right]^{T}, \mathrm{n}=\left[n_{1}, \ldots, n_{l}\right]^{T}, \mathbf{1}=\left[1, \ldots,\left.1\right|^{T}\right.\right.$ and $\operatorname{diag}(x)$ is a diagonal matrix with the elements of vector $x$ along its main diagonal.

The balancing constraint is essential because diag $(\mathrm{V} \cdot \mathbf{1})-\mathrm{V}$ is singular (multiply with 1 to see this). Barring degeneracy, an invertible system of equations can be constructed by replacing any of the $I$ equations in (4.13) by the equation (4.14).

\section{Remark 4.5.}

The criterion (4.3) is very flexible in that it allows one to incorporate a subjective assessment of the seriousness of arbitrage opportunities between different insurers, or groups of insurers.

Take an example. A number of union-based health funds operate in Australia, each of which recruits its membership exclusively from certain occupational groups. It is arguable that arbitrage opportunities between any of these funds are of no concern, as their members cannot easily transfer (although they can transfer to open funds). If desired, an assessment of which arbitrage opportunities actually matter most can be formalised by adjusting appropriately the weights $v_{i j}(x)$. In doing so, however, one must take care to ensure that the matrices $V(x)$ do not become too sparse to allow a unique solution to (4.4).

On a more practical note, care must be taken because an equalisation scheme which bases its allocation on any form of subjective assessment, will be very vulnerable to criticism by the participating insurers, half of which would prefer not to participate in equalisation in the first place.

\section{Remark 4.6.}

With regard to replacing the theoretical quantities $d_{i}(x)$ with suitable estimates $d_{i}^{*}(x)$ and balancing the equalisation scheme against the actual rather than the expected claims cost, the same comments as in section 3 apply.

\section{A NUMERIC EXAMPLE}

This section provides a numeric example to illustrate different equalisation schemes.

Data for 1990-1994 from a random sample of health insurers in Australia was used. The sample was so random, in fact, that the author himself does not know which insurers were chosen; for all intents and purposes the data can be viewed as construed. 
The data, consisting of persons covered and basic benefits paid in each of these years, was split into two age groups, Under $65 \mathrm{~s}$ and Over $65 \mathrm{~s}$. Denote these two groups by $x_{1}$ and $x_{2}$, respectively.

The heterogeneity in benefit plans and thence in the drawing rates $d_{i}(x)$ was modelled and estimated by the credibility method set out in the appendix.

We compared the different equalisation schemes for the year $t=1993$. For insurer $i$, class $x$, we introduce the following notation:

$D_{i}(x)=B_{i}(x) / n_{i}(x) \quad=$ the empirical drawing rate

$d_{i}^{*}(x)=T^{*}(x) \bar{\Theta}_{i}^{*}(x) \quad=$ the credibility-estimated drawing rate;

We further introduce

$D(x)=B(x) / n(x) \quad=$ the empirical drawing rate of class $x$

$d^{*}(x)=\sum_{i=1}^{l} \frac{n_{i}(x)}{n(x)} d_{i}^{*}(x)=$ the credibility-estimated drawing rate of class $x$;

$D_{i}=B_{i} / n_{i} \quad=$ the empirical drawing rate of insurer $i$

$D=B / n \quad=$ the empirical drawing rate across all classes and insurers :

and, finally

$E_{i} \quad=$ the net transfer to equalisation by insurer $i$

$\tilde{D}_{k}=\frac{B_{i}+E_{i}}{n_{i}} \quad=$ the post-equalisation unit cost of insurer $i$.

The following seven equalisation schemes were considered:

\section{a. Schemes which minimise arbitrage against the average}

a.1 The scheme (3.14). The credibility estimators (A.20) of the theoretical drawing rates $d_{i}(x)$ were used. The explicit formula of the scheme is

$$
\tilde{D}_{k}=D+\sum_{x \in \mathrm{X}} p_{k}(x)\left(d_{k}^{*}(x)-d^{*}(x)\right)
$$

a.2 The scheme (3.17). This is essentially the same scheme as in (a.l), except that empirical drawing rates are used to estimate the theoretical drawing rates. The explicit formula of the scheme is

$$
\tilde{D}_{k}=D+\sum_{x \in X} p_{k}(x)\left(D_{k}(x)-D(x)\right)
$$




\section{b. Schemes which minimise arbitrage between insurers}

b.1 The scheme (4.5) with

$$
v_{i j}(x)=\frac{n_{i}(x) n_{j}(x)}{n(x)}
$$

The credibility estimators (A.20) of the theoretical drawing rates $d_{i}(x)$ were used. A matrix inversion was used to solve (4.5).

b.2 The same scheme as in (b.1), but using empirical drawing rates to estimate the theoretical drawing rates.

\section{c. Miscellaneous schemes}

c.1 A simplified version of the 1989 Australian scheme. It is given by the formula

$$
\tilde{D}_{k}=\frac{1}{n_{k}}\left(B_{k}\left(x_{1}\right)+\frac{n_{k}}{n} B\left(x_{2}\right)\right) .
$$

c.2 Full equalisation, given by the formula $\tilde{D}_{k}=D$;

c.3 No equalisation, given by the formula $\tilde{D}_{k}=D_{k}$.

TABLE 5.1

Post-equalisation unit cost $\tilde{D}_{k}$

\begin{tabular}{rccccccc}
\hline \multicolumn{7}{c}{ i } & \multicolumn{7}{c}{ Equalisation scheme } \\
\cline { 2 - 8 } & $\mathrm{a} .1$ & $\mathrm{a} .2$ & $\mathrm{~b} .1$ & $\mathrm{~b} .2$ & $\mathrm{c} .1$ & $\mathrm{c} .2$ & $\mathrm{c} .3$ \\
\hline 1 & 204.68 & 185.74 & 204.37 & 185.29 & 204.17 & 213.25 & 141.17 \\
2 & 200.63 & 206.46 & 200.38 & 206.10 & 219.49 & 213.25 & 171.31 \\
3 & 184.37 & 171.16 & 184.71 & 171.66 & 195.71 & 213.25 & 220.06 \\
4 & 198.28 & 200.94 & 197.94 & 200.43 & 219.95 & 213.25 & 151.22 \\
5 & 224.39 & 226.23 & 224.38 & 226.21 & 219.29 & 213.25 & 224.00 \\
6 & 237.00 & 238.69 & 237.17 & 238.94 & 220.00 & 213.25 & 263.05 \\
7 & 206.64 & 238.58 & 207.04 & 239.16 & 229.11 & 213.25 & 295.90 \\
8 & 202.48 & 199.54 & 202.53 & 199.62 & 205.87 & 213.25 & 207.20 \\
9 & 197.14 & 195.15 & 196.99 & 194.92 & 208.82 & 213.25 & 172.86 \\
10 & 188.85 & 169.98 & 188.57 & 169.57 & 183.30 & 213.25 & 129.87 \\
11 & 207.14 & 198.68 & 206.81 & 198.20 & 218.34 & 213.25 & 152.01 \\
12 & 204.37 & 236.50 & 204.66 & 236.91 & 234.05 & 213.25 & 277.36 \\
\hline
\end{tabular}

We then compared the residual arbitrage under each scheme against the measure (4.3), under the assumption that the credibility estimated drawing rates coincide with the theoretical drawing rates.

For each scheme we calculated the quantity

$S\left(\tilde{D}_{1}, \ldots, \tilde{D}_{l}\right)=\sqrt{\frac{1}{v} \sum_{i=1}^{I} \sum_{j=1}^{I} \sum_{x \in \mathrm{X}} v_{i j}(x)\left(\tilde{D}_{i}-\tilde{D}_{j}-d_{i}^{*}(x)+d_{j}^{*}(x)\right)^{2}}$, 
with $v_{i j}(x)$ defined by (5.3). Scaling (4.3) by $1 / v$ and the square root transformation were chosen in order to obtain numbers on the same scale as the drawing rates.

Obviously the equalisation scheme (b.l) is optimal against this measure. However, we were interested in just how much additional arbitrage is generated by replacing the credibility estimators $d_{i}^{*}(x)$ by empirical drawing rates $D_{i}(x)$, or by using the simpler schemes (a.1) or (a.2).

The results are displayed in Table 5.2 below. We calculated the residual arbitrage for the years 1990-1993.

Table 5.2 indicates that passing from credibility estimates to empirical drawing rates increases the residual arbitrage. There is, however no significant difference between the residual arbitrage in the optimal scheme (b.1) and the simplified scheme (a.1); the same observation holds true for their respective counterparts (b.2) and (a.2). From this one can conclude that the simple model where arbitrage occurs just against the market average, while unrealistic, produces an optimal scheme which is sufficient for practical purposes, at least against the data used in this study.

The 1989 Australian equalisation scheme (c.1) has residual arbitrage in excess of that achieved by the optimal schemes (a.1) and (b.1) and their counterparts (a.2) and (b.2).

Full equalisation and, of course, no equalisation lead to residual arbitrage which is well in excess of what the optimal schemes achieve.

TABLE 5.2

RESIDUAL ARBITRAGE BY SCHEME AND YEAR

\begin{tabular}{lccccccc}
\hline & \multicolumn{7}{c}{ Equalisation scheme } \\
\cline { 2 - 8 } Year & a.1 & a.2 & b.1* & b.2 & c.1 & c.2 & c.3 \\
\hline 1990 & 21.7664 & 25.6775 & 21.7658 & 25.8008 & 27.7776 & 29.3786 & 40.9531 \\
1991 & 25.1756 & 26.8778 & 25.1749 & 26.9551 & 28.8938 & 32.6921 & 43.4677 \\
1992 & 27.4337 & 29.4386 & 27.4327 & 29.5198 & 30.9241 & 35.5532 & 49.3301 \\
1993 & 29.5880 & 31.9841 & 29.5869 & 32.0562 & 34.7802 & 38.1619 & 53.2510 \\
\hline
\end{tabular}

* The scheme b. 1 is optimal by assumption.

\section{ALTERNATIVE MEASUREMENT OF ARBITRAGE}

Some people argue, although not exactly in the words used in this paper, that the measure of arbitrage as in (3.6) or (4.3) is too restrictive. In order to understand their point of view, it is instructive to consider the decomposition of the drawing rate $(3.18)$, repeated here for ease of reference:
$D_{k}=D$
(average drawing rate)

(6.1)

$$
\begin{array}{ll}
+\sum_{x \in \mathrm{X}} p_{k}(x)\left(D_{k}(x)-D(x)\right) & \text { (benefit component) } \\
+\sum_{x \in \mathrm{X}} D(x)\left(p_{k}(x)-p(x)\right) & \text { (profile component) }
\end{array}
$$


The scheme (3.17) can be derived by simply eliminating the profile component while letting each insurer pay for its own benefit component.

The argument put forward by critics of this scheme is that the benefit component isn't all benefits. There are two reasons why this could be the case.

The first reason is that the partition of the insured population into supposedly homogeneous groups may not be fine enough. The cost effect of any differences between different insurers' membership profiles which are not reflected in the partition, will make its way into the benefit component and remain unequalised. Figure 1.1 shows, for instance, that there are significant age-related differences in the cost of insuring people aged more than 60 years. In order to make sure that the effect of these differences is equalised, one will have to define very narrow age bands.

The other reason cited is the insidious effect of self-selection. This means that insures with generous benefit plans are likely to attract a less healthy membership than insurers with no-frills benefit plans. The fact that some insurers aggressively market exclusion plans which are unsuitable for elderly people-excluding hip replacements or bypass operations, for example-even encourages self-selection. It is said in the industry that most people are fairly good judges of their own health care need in the medium term. As a result of self-selection, it is argued, two insurers with identical membership profiles may still have differences in cost which exceed those that can be directly ascribed to differences in their benefit plans.

While the first problem is essentially a statistical one and must be solved in a statistical framework, the problem of self-selection can only be addressed by reviewing our measurement of arbitrage.

Denote the set of all possible treatments which are insurable, by $G$. The treatments in $G$ could be more or less aggregated; at a very fine level of classification, $\mathrm{G}$ could consist of all Diagnostically Related Groups (DRGs).

For an insurer $i \in\{1, \ldots, I\}$, a risk class $x \in \mathrm{X}$, and a treatment $g \in \mathrm{G}$, we define the following quantities:

$H_{i}(x, g)=$ the number of hospital episodes paid for; and

$B_{i}(x, g)=$ the amount of benefits paid.

As before, omission of any argument indicates summation.

Now denote by $a_{i}(x, g)$ the expected benefit cost incurred by insurer $i$ in insuring a person in class $x$ to receive treatment $g$, that is

$$
E\left[B_{i}(x, g) \mid H_{i}(x, g)\right]=H_{i}(x, g) a_{i}(x, g) .
$$

The assumption underlying self-selection is that in choosing an insurer, people compare the overall price with the specific benefits they are likely to receive in return; and, accepting that people are good judges of their own health care need, one may surmise that many self-select quite cynically. We therefore assert that an arbitrage opportunity between insurers $i$ and $j$ exists for people in class $x$ wishing to receive treatment $g$, if

$$
\tilde{d}_{i}-\tilde{d}_{j} \neq a_{i}(x, g)-a_{j}(x, g)
$$

This means that the comparison between different insurers is based on intended usage, rather than the statistical average of benefits received. 
In support of this measure of arbitrage one could also argue (Dubey, 1994) that since the insured person is in no position to know his/her drawing rate, he/she is likely to compare competing insurers by the benefits they offer for specific treatments.

An overall measure of the arbitrage opportunities present in the market would then be

$$
R\left(\tilde{d}_{1}, \ldots, \tilde{d}_{I}\right)=\sum_{i=1}^{I} \sum_{j=1}^{I} \sum_{x \in \mathrm{X}} \sum_{g \in \mathrm{G}} w_{i j}(x, g)\left(\tilde{d}_{i}-\tilde{d}_{j}-a_{i}(x, g)+a_{j}(x, g)\right)^{2},
$$

where

$$
\mathrm{W}(x, g)=\left[\begin{array}{ccc}
w_{11}(x, g) & \ldots & w_{1 I}(x, g) \\
\vdots & \ldots & \vdots \\
w_{I 1}(x, g) & \ldots & w_{I I}(x, g)
\end{array}\right]
$$

are symmetric matrices of fixed, non-negative weights.

Not wanting to entirely abandon the criterion (4.3), we propose to blend the two criteria (4.3) and (6.6) and find an equalisation scheme to solve the constrained minimisation problem

$$
\begin{aligned}
& \text { Minimise } Q\left(\tilde{d}_{1}, \ldots, \tilde{d}_{I}\right)+R\left(\tilde{d}_{1}, \ldots, \tilde{d}_{l}\right) \\
& \text { subject to } \sum_{i=1}^{I} n_{i} \tilde{d}_{i}=n d .
\end{aligned}
$$

By exactly the same technique as in theorem 4.1 we can prove

\section{Theorem 6.1.}

The solution of (6.8) is given by the set of equations

$$
\begin{aligned}
\left(v_{k}+w_{k}\right) \tilde{d}_{k}= & \sum_{i=1}^{I}\left(v_{k i}+w_{k i}\right) \tilde{d}_{i} \\
& +\sum_{x \in \mathrm{X}} v_{k}(x) d_{k}(x)-\sum_{x \in \mathrm{X}} \sum_{i=1}^{I} v_{k i}(x) d_{i}(x) \\
& +\sum_{x \in \mathrm{X}} \sum_{g \in \mathrm{G}} w_{k}(x, g) a_{k}(x, g)-\sum_{x \in \mathrm{X}} \sum_{g \in \mathrm{G}} \sum_{i=1}^{I} w_{k i}(x, g) a_{i}(x, g)
\end{aligned}
$$

for $k=1, \ldots, I$, and

nd $\quad=\sum_{i=1}^{l} n_{i} \tilde{d}_{i}$. 


\section{Remark 6.2.}

The minimum arbitrage scheme can be written in the form

$$
\tilde{d}_{k}=\tilde{d}^{(k)}+\frac{v_{k}}{v_{k}+w_{k}} \Delta_{k}(d)+\frac{w_{k}}{v_{k}+w_{k}} \Delta_{k}(a),
$$

where

$$
\tilde{d}^{(k)}=\sum_{i=1}^{I} \frac{v_{k i}+w_{k i}}{v_{k}+w_{k}} \tilde{d}_{i}
$$

is a weighted average of all insurers' post-equalisation unit cost,

$$
\Delta_{k}(d)=\sum_{x \in \mathrm{X}} \frac{v_{k}(x)}{v_{k}}\left(d_{k}(x)-\sum_{i=1}^{l} \frac{v_{k i}(x)}{v_{k}(x)} d_{i}(x)\right)
$$

is a weighted average of the drawing rate differential between insurer $k$ and the suitably calculated average, and

$$
\Delta_{k}(a)=\sum_{x \in \mathrm{X}} \sum_{g \in \mathrm{G}} \frac{w_{k}(x, g)}{w_{k}}\left(a_{k}(x, g)-\sum_{i=1}^{l} \frac{w_{k i}(x, g)}{w_{k}(x, g)} a_{i}(x, g)\right)
$$

is a weighted average of the benefit differential between insurer $k$ and a suitably calculated average.

Schemes of the form (6.10) can be called mixed schemes, as they minimise arbitrage opportunities with respect to a mixed criterion. In the extreme case where $v_{k}=0(\forall k)$, the resulting equalisation scheme can be called a usage scheme, as it minimises arbitrage opportunities based on intended usage only. The scheme proposed for Ireland is a usage scheme, although it does not differentiate between different treatments.

\section{Corollary 6.3.}

If the matrix $\mathrm{V}+\mathrm{W}=\sum_{x \in \mathrm{X}} \mathrm{V}(x)+\sum_{x \in \mathrm{X}} \sum_{g \in \mathrm{G}} \mathrm{W}(x, g)$ satisfies the condition

$$
v_{i j}+w_{i j}=c n_{i} n_{j}
$$

with an arbitrary constant $c>0$, then optimal scheme against the criterion (6.8) has an explicit solution.

\section{Remark 6.4.}

With regard to replacing the theoretical quantities $d_{i}(x)$ with suitable estimates $d_{i}^{*}(x)$ and balancing the equalisation scheme against the actual rather than the expected claims cost, the same comments as in section 3 apply. However, to implement the equalisation scheme $(6.10)$, one also needs estimates of the quantities $a_{i}(x, g)$; if benefits are paid in the form of predetermined case payments, such estimates are readily available. 


\section{PARTIAL COMMUNITY RATING}

In sections 3 and 4 we developed equalisation schemes which were designed to support total community rating, i.e. just one flat contribution rate for any person/policy.

Some countries/states require partial community rating. As an example of partial community rating we will use unisex rating in motor vehicle insurance.

By the argument developed in section 2 , mandatory partial community rating can imply an obligation to implement an equalisation scheme, just as total community rating. A pragmatic test as to whether equalisation is warranted would be to consider the difference in cost between hypothetical insurers located at the extremes of membership profile: if for instance an insurer which has recruited only males has significantly higher cost than one which has recruited only females, other things being equal, then equalisation is warranted. Following this argument further, one can conjecture that equalisation normally will be warranted whenever partial community rating is mandatory; for unless there were significant differences in cost, there would be no need to make partial community rating mandatory.

In this section we develop an equalisation scheme that supports partial community rating.

As before, assume that there exists a partition $X$ of the insured population and that contribution rates may not depend on $x \in X$. The partition could be $X=\{$ male, female\}, for instance. Now assume that there exists another partition $Y$, and that contribution rates may differ with $y \in \mathrm{Y}$; this partition may be made up of several other rating variables, like the type of car, district, usage, etc. Thus we assume that each insurer $i$ is allowed to have a vector of (net) contribution rates,

$$
\tilde{\mathrm{d}}_{\mathrm{i}}=\underset{y \in \mathrm{Y}}{\operatorname{col}}\left(\tilde{\mathrm{d}}_{i}(y)\right)
$$

Denote by $d_{i}(x, y)$ the pure premium of insurer $i$ in insuring a person belonging to the class $(x, y) \in \mathrm{X} \times \mathrm{Y}$.

We say that an opportunity for arbitrage exists if for insurers $i, j \in\{1, \ldots, I\}$ and risk classes $x \in \mathrm{X}, y, y^{\prime} \in \mathrm{Y}$ we have

$$
\tilde{d}_{i}(y)-\tilde{d}_{j}\left(y^{\prime}\right) \neq d_{i}(x, y)-d_{j}\left(x, y^{\prime}\right)
$$

This measure implies that

a. for a person in class $(x, y) \in \mathrm{X} \times \mathrm{Y}$, an arbitrage opportunity exists when there is a mismatch between the contributions and benefits between the two insurers; and

b. for a person in class $x \in \mathrm{X}$, an arbitrage opportunity exists if there is a mismatch between contributions and benefits for two values $y, y^{\prime} \in \mathrm{Y}$, be it with the same insurer or a different insurer.

Arbitrage of type (a) is of the form we have already seen previously, between insurers. The motivation for including (b) is that in transferring between classes $y$ and $y^{\prime}$ (say, changing cars), the change in contribution rate for a person $x$ should 
reflect the change in pure premium for class $x$. The change in contribution rate should not reflect the fact that car make $y$ appeals more to males (females) than car $y^{\prime}$. If contributions for $y^{\prime}$ were much higher than those for $y$ on account of a different mix of $x$, the purpose of partial community rating-equality between classes of $\mathrm{X}$-would be defeated. Barred from discriminating on the basis of $x$, insurers would surely find proxy variables to include in $y$.

Having motivated what we mean by arbitrage, we can define an overall measure

(7.3) $Q\left(\tilde{d}_{1}, \ldots, \tilde{d}_{l}\right)=$

$$
\sum_{i, j=1}^{I} \sum_{x \in \mathrm{X}} \sum_{y, y^{\prime} \in \mathrm{Y}} v_{i j}\left(x ; y, y^{\prime}\right)\left(\tilde{d}_{i}(y)-\tilde{d}_{j}\left(y^{\prime}\right)-d_{i}(x, y)+d_{j}\left(x, y^{\prime}\right)\right)^{2},
$$

where the $v_{i j}\left(x ; y, y^{\prime}\right)$ are non-negative weights obeying the symmetry condition $v_{i j}\left(x ; y, y^{\prime}\right)=v_{j i}\left(x ; y^{\prime}, y\right)$.

An optimal equalisation scheme against the measure (7.3) is given by the set of values $\left\{\tilde{d}_{i}(y) \mid i=1, \ldots, I ; y \in \mathrm{Y}\right\}$ which minimises (7.3) under the constraint

$$
\sum_{i=1}^{l} \sum_{y \in \mathrm{Y}} n_{i}(y) \tilde{d}_{i}(y)=\sum_{i=1}^{l} \sum_{x \in \mathrm{X}} \sum_{y \in \mathrm{Y}} n_{i}(x, y) d_{i}(x, y)=: n d \text {. }
$$

Using Lagrange minimisation we obtain

\section{Theorem 7.1.}

The minimum arbitrage scheme against the criterion (7.3) is given by the set of linear equations

$$
\begin{aligned}
v_{k} \cdot(\cdot ; z, \cdot) \tilde{d}_{k}(z) & =\sum_{i=1}^{I} \sum_{y \in \mathrm{Y}} v_{k i}(\cdot ; z, y) \tilde{d}_{i}(y) \\
& +\sum_{x \in \mathrm{X}} v_{k}(x ; z, \cdot) d_{k}(x, z) \\
& -\sum_{i=1}^{I} \sum_{x \in \mathrm{X}} \sum_{y \in \mathrm{Y}} v_{k i}(x ; z, y) d_{i}(x, y)
\end{aligned}
$$

for $k=1, \ldots, I$ and $z \in Y$, and

$$
\sum_{k=1}^{l} \sum_{z \in \mathrm{Y}} n_{k}(z) \tilde{d}_{k}(z)=n d
$$

where a dot in place of an argument indicates summation.

Proof: Lagrange minimisation, 


\section{Remark 7.2.}

The system of equations (7.5)-(7.6) has an explicit solution if the following condition holds :

$$
v_{k i}(\cdot ; z, y)=c \cdot n_{k}(z) n_{i}(y) \text {, with } c \text { an arbitrary constant. }
$$

\section{CONCLUDING REMARKS}

This paper provides a theoretical framework for the development of equalisation schemes, be it in health insurance or other areas. Broadly, it argues that community rating creates arbitrage opportunities which are self-reinforcing and can destabilise a market. As a consequence, an equalisation scheme which is meant to support community rating, must be designed to minimise opportunities for arbitrage.

The use of theoretically sound equalisation schemes may help to bridge the gap between the advocates of unrestricted risk rating and the advocates of (partial) community rating, which is lucidly described in Jewell (1980).

The general approach advocated in this paper still leaves some degree of freedom to the designer. In particular, the measure used to quantify arbitrage could be varied, although the measure proposed here (weighted sum of squares) has the great advantage of being mathematically tractable. Even when retaining a measure based on weighted sum of squares, one can vary the weights which indicate the "seriousness" of different arbitrage opportunities.

The weighted sum of squares measure leads to equalisation formulae which are very tractable and, to the trained eye, intuitively obvious. It is the author's hope that this paper will contribute towards a more disciplined approach to the construction of equalisation schemes.

With effect from 1995 the Australian equalisation scheme was modified following the recommendations in MIRA $(1993,1994)$. The new scheme combines features of the composition-based scheme (3.17) and the 1989 Australian scheme (5.4).

\section{ACKNOWLEDGEMENTS}

This paper developed out of a study commissioned by the Australian Department of Health, Housing and Community Services in 1992. Many persons within the Department in the health insurance industry have contributed to the ideas presented above. All contributions are gratefully acknowledged, in particular those of Malcolm Murray (Health Insurance Commission) and Russell Schneider (Australian Health Insurance Association) and Ian Heppell (then MIRA Consultants Ltd). 
The heterogeneity in benefit plans and thence in the pure premiums $d_{i}(x)$ was modelled as follows: It was assumed that the relative level of benefits paid by insurer $i$ is characterised by a latent vector

$$
\Theta_{i}=\underset{x \in \mathrm{X}}{\operatorname{col}}\left(\theta_{i}(x)\right)
$$

which is independent of time. The notation col denotes a column vector.

We then assumed that the pure premium vector for insurer $i$ in year $t$ is

$$
\mathrm{d}_{i}^{(t)}=\operatorname{col}_{x \in \mathrm{X}}\left(d_{i}^{(t)}(x)\right)=\underset{x \in \mathrm{X}}{\operatorname{col}}\left(T^{(t)}(x) \theta_{i}(x)\right)=\mathrm{T}^{(t)} \Theta_{i},
$$

where $\mathrm{T}^{(t)}=\underset{x \in \mathrm{X}}{\operatorname{diag}}\left(T^{(t)}(x)\right)$ and $T^{(t)}(x)$ adjusts benefits paid to class $x$ in year $t$

to reflect inflation.

As an aside, note that the adjustment $T^{(t)}$ could be chosen dependent on the insurer $i$, thereby incorporating in the model any prior knowledge about the relative benefit levels provided by the different insurers. We have preferred not to use this option and rather let the latent vector $\Theta_{i}$ reflect all the variation in benefits between insurers.

Of actual benefits paid by insurer $i$ in year $t$ we assumed that

$$
\mathrm{E}\left[B_{i}^{(t)}\right]=\underset{x \in \mathrm{X}}{\operatorname{col}}\left(\mathrm{E} B_{i}^{(t)}(x)\right)=\operatorname{col}_{x \in \mathrm{X}}\left(n_{i}^{(t)}(x) d_{i}^{(t)}(x)\right)=\mathrm{N}_{i}^{(t)} \mathrm{T}^{(t)} \Theta_{i}
$$

where $\mathrm{N}_{i}^{(t)}=\underset{x \in \mathrm{X}}{\operatorname{diag}}\left(n_{i}^{(t)}(x)\right)$. Of the variance we assumed

$$
\operatorname{Var}\left[B_{i}^{(t)}\right]=\underset{x \in \mathrm{X}}{\operatorname{diag}}\left(T^{(t)^{2}}(x) n_{i}^{(t)}(x) \Phi(x)\right)=\mathrm{T}^{(t)^{2}} \mathrm{~N}_{i}^{(t)} \Phi
$$

independent of $\Theta_{i}$, where $\Phi=\underset{x \in \mathrm{X}}{\operatorname{diag}}(\Phi(x))$ is a fixed coefficient matrix.

Finally we assumed that $\Theta_{1}, \ldots, \Theta_{l}$ are independent random vectors with mean

$$
\mathrm{E} \Theta_{i}=\eta
$$

and variance

$$
\operatorname{Var} \Theta_{i}=\Lambda
$$


The best linear estimator of $\Theta_{i}$, homogeneous in the $B_{i}^{(t)}$, is

$$
\bar{\Theta}_{i}=Z_{i} \hat{\Theta}_{i}+\left(I-Z_{i}\right) \hat{\eta},
$$

where

$$
\hat{\Theta}_{i}=\left(\sum_{t} \mathbf{N}_{i}^{(t)}\right)^{-1} \sum_{t} \mathbf{N}_{i}^{(t)} \hat{\Theta}_{i}^{(t)}
$$

and

$$
\hat{\Theta}_{i}^{(t)}=\left[\mathrm{N}_{i}^{(t)} \mathrm{T}^{(t)}\right]^{-1} \mathbf{B}_{i}^{(t)},
$$

are estimators of $\Theta_{i}$,

$$
\mathrm{Z}_{i}=\Lambda\left(\mathrm{N}_{i}^{(\cdot)^{-1}} \Phi+\Lambda\right)^{-1}
$$

is the credibility matrix (the dot denoting summation), and finally

$$
\hat{\eta}=\left(\sum_{i=1}^{I} Z_{i}\right)^{-1} \sum_{i=1}^{l} Z_{i} \hat{\Theta}_{i}
$$

is the best linear unbiased estimator of $\eta$.

It now remains to estimate the parameters of the model. The parameters $T^{(t)}(x)$ were estimated by

$$
T^{(t)^{*}}(x)=\frac{B^{(t)}(x) / n^{(t)}(x)}{B^{(1994)}(x) / n^{(1994)}(x)},
$$

and the resulting estimate inserted in (A.9).

The structural parameters were estimated by the method proposed by de Vylder (1981) and analysed by Hesselager (1988).

The covariance matrix $\Phi$ was estimated by the unbiased estimator

$$
\Phi^{*}=\operatorname{diag}_{x \in \mathrm{X}}\left(\frac{1}{I} \sum_{i=1}^{I} \frac{1}{\tau_{i}-1} \sum_{t} n_{i}^{(t)}(x)\left(\hat{\Theta}_{i}^{(t)}(x)-\hat{\Theta}_{i}(x)\right)^{2}\right) .
$$

In this expression, $\boldsymbol{\tau}_{i}$ represents the number of years that the insurer $i$ has been under observation.

The covariance matrix $\Lambda$ was estimated by the limit of a convergent sequence of the form

$$
\Lambda^{*}(k+1)=\frac{1}{I-1} \sum_{i=1}^{I} Z_{i}^{*}(k)\left(\hat{\Theta}_{i}-\hat{\eta}(k)\right)\left(\hat{\Theta}_{i}-\hat{\eta}(k)\right)^{T},
$$

with

$$
\mathrm{Z}_{i}^{*}(k)=\Lambda^{*}(k)\left(\mathrm{N}_{i}^{(\cdot)^{-1}} \Phi^{*}+\Lambda^{*}(k)\right)^{-1}
$$


and

$$
\hat{\boldsymbol{\eta}}(k)=\left(\sum_{i=1}^{l} \mathrm{Z}_{i}^{*}(k)\right)^{-1} \sum_{i=1}^{l} \mathrm{Z}_{i}^{*}(k) \hat{\Theta}_{i} .
$$

Denoting the limit by $\Lambda^{*}(\infty)$, the final estimator used was the symmetrised

$$
\Lambda^{* *}=\frac{1}{2}\left[\Lambda^{*}(\infty)+\Lambda^{*}(\infty)^{T}\right]
$$

Using $\Lambda=\mathrm{I}$ (identity matrix) as starting value, this procedure worked extremely well.

The resulting estimates were

$$
\mathrm{T}^{(1990, \ldots 1994)^{*}}=\left[\begin{array}{lllll}
0.92 & 0.92 & 0.98 & 0.99 & 1.00 \\
0.76 & 0.86 & 0.92 & 0.95 & 1.00
\end{array}\right]
$$

and

(A.19) $\hat{\eta}=\left[\begin{array}{l}143.08 \\ 730.37\end{array}\right], \quad \Phi^{*}=\left[\begin{array}{rr}3185^{2} & 0 \\ 0 & 6156^{2}\end{array}\right], \quad \Lambda^{* *}=\left[\begin{array}{rr}14.38 & 49.31 \\ 49.31 & 644.18\end{array}\right]$

We denote the credibility estimator of the form (A.7), with the parameter values (A.19) inserted, by

$$
\bar{\Theta}_{i}=\operatorname{col}_{x \in X}\left(\bar{\theta}_{i}^{*}(x)\right)
$$

Note in passing that inflation apparently has been significantly stronger for the Over $65 \mathrm{~s}$ than the Under 65s, see (A.18). There are two possible explanations for this. One explanation is that the 1989 equalisation scheme essentially has removed any incentive for insurers to control benefits paid to that group. The other possible explanation is that there has been an ageing in that group; a glance at Figure 1.1 reveals that increasing the average age with just a few years would lead to a significant increase in cost. We did not have the data available to decide the extent to which each of the two explanations outlined above explain the observed difference in inflation.

The estimates $\bar{\Theta}_{i}^{*}$ are shown in Table A.1. Based on these estimates one can estimate the pure premium vector of insurer $i$ in year $t$ as

$$
\mathrm{d}_{i}^{(t)^{*}}=\mathrm{T}^{(t)^{*}} \bar{\Theta}_{i}^{*}=\underset{x \in X}{\operatorname{col}}\left(T^{(t)^{*}}(x) \bar{\theta}_{i}^{*}(x)\right)
$$


TABLE A.1

Credibility estimates

\begin{tabular}{rrrrrrr}
\hline \hline$i$ & $\begin{array}{c}n_{i}^{(\cdot)}\left(x_{1}\right) \\
\text { in } 1000\end{array}$ & $\begin{array}{c}n_{i}^{(\prime \prime}\left(x_{2}\right) \\
\text { in } 1000\end{array}$ & $\hat{\Theta}_{i}\left(x_{1}\right)$ & $\hat{\Theta}_{i}\left(x_{2}\right)$ & $\bar{\Theta}_{i}^{*}\left(x_{1}\right)$ & $\hat{\Theta}_{i}^{*}\left(x_{2}\right)$ \\
\hline 1 & 95 & 4 & 127.56 & 583.65 & 140.71 & 716.46 \\
2 & 247 & 13 & 128.86 & 616.84 & 138.35 & 700.57 \\
3 & 150 & 31 & 160.78 & 501.55 & 140.84 & 660.28 \\
4 & 781 & 20 & 131.05 & 515.07 & 134.94 & 665.50 \\
5 & 1,996 & 232 & 157.11 & 851.67 & 155.06 & 831.16 \\
6 & 2,653 & 416 & 160.95 & 904.53 & 159.36 & 885.49 \\
7 & 314 & 73 & 172.72 & 724.58 & 151.06 & 741.92 \\
8 & 2,223 & 284 & 143.58 & 725.78 & 143.35 & 726.99 \\
9 & 1,611 & 115 & 134.38 & 689.03 & 136.67 & 696.97 \\
10 & 578 & 26 & 105.52 & 686.52 & 126.35 & 676.33 \\
11 & 55 & 2 & 143.22 & 559.64 & 142.73 & 725.48 \\
12 & 126 & 24 & 177.17 & 709.42 & 147.51 & 737.24 \\
\hline
\end{tabular}

\section{REFERENCES}

Department of Health of THE Republic of Ireland (1994). Briefing Paper on Health Insurance Regulatory Framework in Ireland.

De WIT, G.W. and VAN EEGHEN, J. (1984). Rate making and society's sense of faimess. ASTIN Bulletin 14, 151-163.

DE VYLDER, F. (1981). Practical credibility theory with emphasis on optimal parameter estimation. ASTIN Bulletin 12, 115-131.

DubeY, A. (1994). Personal communication.

GreGORIUS, F.K. (1987). The insurance of hospitalisation and sickness in the Netherlands. Paper presented to the XX ASTIN Colloquium, Scheveningen.

HESSELAGER, O. (1988). Estimation of variance components in hierarchical regression models with nested classification. Working paper No. 69 from the Laboratory of Actuarial Mathematics, University of Copenhagen.

Jewell, W.S. (1980). Models in Insurance: Paradigms, Puzzles, Communications and Revolutions. Transactions of the 21st International Congress of Actuaries.

Neuhaus, W. (1995). Balanced Credibility Estimation. Working Paper No. 127 from the Laboratory of Actuarial Mathematics, University of Copenhagen.

MACINTYRE, D.M. (1962). Voluntary Health Insurance and Rate Making. Cornell University Press.

MIRA Consultants Ltd. (1993). Review of the Private Health Insurance Reinsurance Arrangements. Report prepared for the Department of Health, Housing and Community Services, Canberra.

MIRA CONSULTANTS Ltd. (1994). The Reinsurance Follow-Up Study. Report prepared for the Department of Human Services and Health, Canberra.

Thomson, P. (1994). Personal communication.

\section{Walther NeuHaUs}

Laboratory of Actuarial Mathematics, University of Copenhagen

Universitetsparken 5, DK-2100 Copenhagen $\emptyset$ 